\title{
Dynamic Coherence in Excitonic Molecular Complexes under Various Excitation Conditions
}

\author{
Aurélia Chenu, Pavel Malý and Tomáš Mančal \\ Faculty of Mathematics and Physics, Charles University in Prague, \\ Ke Karlovu 5, 12116 Prague 2, Czech Republic
}

\begin{abstract}
We investigate the relevance of dynamic quantum coherence in the energy transfer efficiency of molecular aggregates. We contrast the dynamics after excitation of a quantum mechanical system with that of a classical system. We demonstrate how a classical description of an ensemble average can be satisfactorily interpreted either as a single system driven by a continuous force or as an ensemble of systems each driven by an impulsive force. We derive the time evolution of the density matrix for an open quantum system excited by light or by a neighboring antenna. We argue that unlike in the classical case, the quantum description does not allow for a formal decomposition of the dynamics into sudden jumps in the quantum mechanical state. Rather, there is a natural finite time-scale associated with the excitation process. We propose a simple experiment how to test the influence of this time scale on the yield of photosynthesis. Because photosynthesis is intrinsically an average process, the efficiency of photosynthesis can be assessed from the quantum mechanical expectation value calculated from the second-order response theory, which has the same validity as the perturbative description of ultrafast experiments. We demonstrate using typical parameters of the currently most studied photosynthetic antenna, the Fenna-Matthews-Olson (FMO) complex, and a typical energy transfer rate from the chlorosome baseplate, that dynamic coherences are averaged out in the complex despite excitation proceeding through a coherent superposition of its eigenstates. The dynamic coherence averages out even when the FMO model is completely free of all dissipation and dephasing. We conclude that under natural excitation conditions, coherent dynamics cannot be responsible for the remarkable efficiency of the photosynthesis, even when considering the dynamics at a single molecular level.
\end{abstract}

\section{INTRODUCTION}

In the light-harvesting antennae of both plants and bacteria and in other molecular aggregates and crystals, electronic excited state delocalization has been since long time ago known to play a significant role in establishing their energy transport properties (e.g. [1-4]). Delocalization of the electronic eigenstates over more than one pigment corresponds to a correlation between electrons of different pigments in the same molecular crystal or aggregate which results from their direct mutual electrostatic interaction. Correspondingly, this effect has been often referred to as electronic quantum coherence. In photosynthetic antennae, the excited eigenstate delocalization enables fast transfer of excitation in space and, in combination with the influence of the virtually infinite number of degrees of freedom (DOF) of the protein environment, directs the energy flow in a funnel like fashion towards the energetically lower lying states of the photosynthetic reaction center $(\mathrm{RC})$. Here the chemical part of the photosynthesis starts. The several order of magnitude difference in the energy transfer rates and the rates of the excitation recombination in the antenna (inverse picoseconds vs. inverse nanoseconds, respectively) explains then the remarkable efficiency of the photosynthetic antennae. Because light interacts resonantly with the electronic excited states, and because the wavelength of the light is usually several orders of magnitude larger than the photosynthetic antennae, the light excites the delocalized eigenstates rather than the individual molecules themselves. This invites a kinetic picture in which the antenna is represented by a set of excited states with energy relaxation between them. The ultra short laser light pulses used in laboratory experiments inevitably excite superpositions of these antenna eigenstates, because they spectrally cover several of them. Nevertheless, the kinetic picture usually assumes independence of the oscillating coherent components of the corresponding electronic density matrix from the much slower varying population components. The presence of any coherence induced by lasers has thus no bearing on the energy transfer in this picture. This assumption is usually termed secular approximation.

With the advent of the two-dimensional (2D) coherent Fourier transformed spectroscopy [5 7], a technique became available which could detect the time-dependent signatures of the superpositions of the antenna excited eigenstates. The properties of the oscillating signatures were predicted for the photosynthetic Fenna-MatthewsOlson (FMO) pigment-protein complex (PPC) [8], and the oscillations were soon observed experimentally [9]. Similar oscillations were apparently observed even earlier on the same system in Ref. [10], but it was only with the availability of the new spectroscopic technique that the effect received widespread attention. Similar oscillations have since been observed in other photosynthetic complexes such as the RC of a purple bacteria [11], the light-harvesting complex II (LHCII) from higher plants [12, 13], from marine algae [13, 14] and from purple bacteria [15] at room temperature (RT), as well as in conjugated polymers [16]. Somewhat unfortunately, this transient oscillatory effect was consistently referred to as elec- 
tronic quantum coherence, i.e. with the term previously assigned to excited state delocalization. In order to lift the overloading of the term quantum coherence, we will refer throughout this paper only to the quantum beats observed by the 2D spectroscopy as quantum coherence. Excited state delocalization is a well established, almost self explanatory term, and it thus justifies dropping the earlier meaning of the term quantum coherence. Dynamic coherence does not require delocalization, nor vice versa. In general, these two effects are entirely unrelated. Delocalization has, however, an effect on the life-time of the dynamic coherence, once it is created in the system. A recent study which clearly distinguishes the different phenomena currently termed quantum coherence in the literature and argues that only exciton delocalization can have an effect on energy transfer is e.g. Ref. [17].

One remarkable deviation from the early predictions about the coherent oscillations in FMO was their unexpectedly long life-time, which reaches beyond 1 ps. It was immediately speculated that the surprisingly long lifetime of the oscillations reflects some intrinsic dynamic quantum mechanical behavior of the photosynthetic antennae, which is crucial in achieving their high efficiency. Table I presents the measured life-times of the observed quantum beats in the different photosynthetic complexes. These oscillations have been mostly interpreted as a signature of superpositions of electronic eigenstates. While in some cases, their life-time is only few hundreds of femtoseconds [14, 15, 18], several photosynthetic complexes exhibit surprisingly long-lived oscillations $[9,11-13,18-$ 20]. A variety of theoretical models assuming an electronic origin have been considered, and despite the diversity of approaches, they predict oscillations with similarly short life-time, i.e. some hundreds of fs (see [21], references therein, and also [22 24]). Although it is sometimes argued that this life-time is 'long', it is still well below the ps time scale observed in a number of experiments at low temperature (e.g. 20]), where oscillations still persist at the end of the measurement time limit. It is important to note that the expected life-time of the oscillations in FMO, at the time of their first measurements, was based on the estimate of Ref. [8], which used the relaxation rates calculated in Ref. [25]. According to Ref. [8], $100-300$ fs is a characteristic value for the coherence life-time, and for the coherence between e.g. the two lowest energy excitons, the estimated life-time is $\tau_{12} \approx 540 \mathrm{fs}$, based on the rates calculated in Ref. [25]. It is with respect to this life-time that the oscillations measured in Ref. [9] were considered long. In all these theoretical estimates, the effects of delocalization are fully taken into account, yet the predicted life-times of coherence are remarkably shorter than the measured values. This invalidates the argument that the measured dynamic coherence is somehow an experimental measure of eigenstate delocalization.

The main mechanism currently suspected to extend the electronic coherence life-time over a ps assumes spatial correlation between environmental degrees of free- dom (DOF) of different pigments [11, 26 34]. Such correlation was however not confirmed by molecular dynamics calculations [35, 36]. Recent theories based on resonance coupling between the electronic and vibrational states [37 40] successfully predict picosecond life-time. It should be noticed that this model, by considering explicitely vibrational DOF of the molecular system, effectively introduces correlation between the bath of different states on the same pigment. As such, it is in line with the assumption of correlated environmental DOF without contradiction with results from molecular dynamics simulations.

The observation of quantum beating in various complexes seems to suggest that this is a general phenomenon in photosynthetic systems under the laboratory excitation conditions. But whether these effects are of importance for the high efficiency of natural photosynthesis or whether they are just artifacts of the ultra fast excitation used in laboratory situation remains a disputed question. As inferred by the authors from the current debate, it is often assumed that the dynamic coherence plays an essential role in light-harvesting efficiency. Such an assumption rests on the notion that a time dependent effect, similar to the one observed in time-resolved experiments with ultra fast pulses, also accompanies light-harvesting of natural sunlight. Suggestions have been made that a thermal source consists of a collection of random femtosecond pulses, and that the experimental observations would be representative of the in vivo conditions [41]. However, based on both semiclassical and fully quantum mechanical representation of the light-matter interaction, it has been demonstrated that excitation by incoherent light as well as one-photon absorption lead to a stationary mixed state, and cannot create a superposition of energy eigenstates (i.e. dynamic coherence), neither on isolated molecules [42, 43] nor on open systems [44, 45]. Thereby, the existence of quantum coherence has been shown to be dependent on the excitation process [43, 44], which is in contrast with a qualitative description suggesting that the absorption of a single photon triggers the same coherent molecular response, regardless of the character of the light source [46].

We are apparently left with two pictures supposedly explaining efficiency of the EET in photosynthesis. The earlier kinetic picture in which coherent oscillations, although present, have no influence on the energy transfer, and the newer coherent picture, in which coherence is posited to have a crucial role. It is thus either the interplay of delocalization with the environment or the interplay of quantum coherence (in the above defined sense) with the environment which lead to high efficiency of the photosynthesis. One might suggest a combined picture, in which the effect of coherence plays a role of a correction to the kinetic picture. In this case the influence of coherence would occur through the coupling between the oscillating terms of the density matrix and the eigenstate population elements, going thus beyond the secular approximation. Such coupling was indeed observed 
in Ref. [20]. The relative contribution of this effect to the EET dynamics remains a question. Predictions using the secular approximation already lead to a close to unity efficiency of the energy transfer, and it is therefore questionable that the improvement on the order of several per cent would play a role for natural photosynthesis, especially considering that it is often even required to decrease the efficiency drastically to avoid damage by over-excitation. There is so-far no evidence that nonsecular effects play any significant role in the EET in photosynthetic antennae.

The aim of this paper is to clarify the relation between the dynamics induced by an ultrafast excitation and the one corresponding to a slow driving force. In particular we will attempt to shed light on the time-scale on which excitation of a molecular system occurs when it is driven by an external source of excitation, such as light or a neighboring antenna. In Section $\Pi$ we will discuss certain equivalence between a slowly driven dynamics of a single classical system and an average over time-evolution of an ensemble of classical systems excited impulsively. In Section [II] we formulate the theory of the excitation the molecular system by light, and in Section IV we use the same formulation to describe an excitation transfer from another molecular system. We discuss the time-scale of the excitation events in Section $\nabla$ and we propose a simple experiment which could test the influence of this time scale on the yield of photosynthesis. We demonstrate the properties of the excited state of a model antenna under excitation feeding from a neighboring system in Section VI. Our conclusions are presented in Section VII

\begin{tabular}{|c|c|c|cr|}
\hline Measured system & Exp. tech. & Temp. & Life time & \\
\hline \hline FMO (Chlorobium tepidum) & 2D ES & $77 \mathrm{~K}$ & $>660 \mathrm{fs}$ & {$[9]$} \\
\hline & & $277 \mathrm{~K}$ & $>300 \mathrm{fs}$ & {$[19]$} \\
\hline & & $77 \mathrm{~K}$ & $>1800 \mathrm{fs}$ & {$[20]$} \\
\hline RC (Rhodobacter Sphaeroides) & 2CECPE & $77 \mathrm{~K}$ & $440 \mathrm{fs}$ & {$[11]$} \\
\hline & & $180 \mathrm{~K}$ & $310 \mathrm{fs}$ & {$[11]$} \\
\hline LH2 (Rhodobacter Sphaeroides) & 2D ES & RT & $400 \mathrm{fs}$ & {$[15]$} \\
\hline LH2 (purple bacteria) & ARC WM & RT & $>2800 \mathrm{fs}$ & {$[13]$} \\
\hline LHCII (Arabidopsis Thaliana) & 2D ES & $77 \mathrm{~K}$ & $>500 \mathrm{fs}$ & {$[12]$} \\
\hline PC645 and PE545 (marine cryptophytes) & 2D PES & $294 \mathrm{~K}$ & $>130 \mathrm{fs}$ & {$[14,18]$} \\
\hline
\end{tabular}

Table I: Characteristics of the quantum beats observed in various photosynthetic systems by different experimental techniques: 2D Electronic Spectroscopy (2DES), 2D Photo Echo Spectroscopy (2DPES), Two-Color Electronic Coherence Photon Echo (2CECPE) and angle-resolved coherent wave mixing (ARC WC). The sign "> means that the coherence life-time was estimated to be larger than the given value.

\section{RELATION BETWEEN IMPULSIVE AND SLOWLY DRIVEN DYNAMICS IN CLASSICAL SYSTEMS}

The relation between the dynamics observed in an ultrafast excitation by a pulsed laser and the one which occurs when the system is driven by a weak steady illumination can be illustrated on a completely classical example of driving the dynamics of a classical system. Even in a laboratory experiment with laser pulses, the excited state dynamics of molecular systems relevant for photosynthesis can be described by introducing a source term into the reduced density matrix equations. In the density matrix formalism, this term is linear in excitation field intensity (i.e. quadratic in electric field). Validity of the description by such linear term in intensity is the same as the third order perturbation theory description of the four-wave mixing (FWM) experiments, such as the 2D Fourier transformed spectroscopy. After a molecular system is excited in a FWM experiment by two elemen- tary light-matter interactions ( $2^{\text {nd }}$ order), there is only one more excitation field acting linearly and producing the stimulated signal [47]. This signal can be experimentally verified to be of the third order in field. Second order treatment of the laboratory and Sun light excitation is therefore completely satisfactory. When treated in a wavefunction formalism, the source term can even be linear in the exciting field, because the calculated observable is quadratic in the wavefunction.

To illustrate the relation between the dynamics of a system driven by a short external impulsive force and a system driven by a slowly varying force, let us consider a classical oscillator which is driven by an external force $f(t)$. The corresponding equations of motion read

$$
\begin{gathered}
\dot{p}=-\omega q+f(t) \\
\dot{q}=+\omega p .
\end{gathered}
$$

This problem can be rewritten formally into the form

$$
\dot{\phi}(t)-H \phi(t)=\varphi_{0} f(t),
$$


where $\phi(t)=\left(\begin{array}{l}p \\ q\end{array}\right)$ represents the phase-space vector consisting of the oscillator coordinate and its conjugated momentum, $\varphi_{0}=\left(\begin{array}{l}0 \\ 1\end{array}\right)$ ensures that $f(t)$ drives only the momentum, and $\mathrm{H}=\left(\begin{array}{cc}0 & +\omega \\ -\omega & 0\end{array}\right)$. The solution of the full equation, Eq. (2), can be found by first finding the solution of the same equation, but with an "ultrashort" driving term on its right hand side (r.h.s.), so-called particular solution $\phi_{\delta}(t)$. For this we have

$$
\dot{\phi}_{\delta}(t)-H \phi_{\delta}(t)=\varphi_{0} \delta(t) .
$$

The particular solution of this equation can be written in form of a Green's function $G(t)$ as

$$
\phi_{\delta}(t)=G(t) \varphi_{0}=\Theta(t)\left(\begin{array}{c}
\sin \omega t \\
\cos \omega t
\end{array}\right),
$$

where

$$
G(t)=\Theta(t) \exp \{H t\}=\Theta(t)\left(\begin{array}{cc}
\cos \omega t & \sin \omega t \\
-\sin \omega t & \cos \omega t
\end{array}\right),
$$

and $\Theta(t)$ is the Heaviside step function. A reaction of the oscillator to the "shock-like" ultrashort excitation is an oscillatory motion. In case of a general driving force, the evolution of the phase-space vector can be shown to be given by

$$
\phi(t)=\int_{0}^{\infty} \mathrm{d} \tau G(t-\tau) \varphi_{0} f(\tau)
$$

which indicates that the dynamics of a system under any type of excitation can be found by averaging in time over the transient impulsively excited dynamics with the weight given by the driving function $f$. This includes even the situation when the driving force is constant, $f(t)=f_{0}$. In this case, the solution can be found intuitively to be a stationary displaced oscillator

$$
\phi_{f_{0}}(t)=\left(\begin{array}{c}
\frac{f_{0}}{\omega} \\
0
\end{array}\right) .
$$

The same result can be obtained from Eq. (6) by assuming that sometime in the past, the function $f(t)$ was slowly switched on in such a way that $\frac{d}{d t} f(t) \approx 0$ at all times. The function became constant, $f(t)=f_{0}$, at some later time. Substituting the definitions of all quantities into Eq. (6), using $\tau^{\prime}=t-\tau$ and integrating by parts, we get

$$
\begin{aligned}
\phi(t) & =\int_{0}^{t} \mathrm{~d} \tau^{\prime}\left(\begin{array}{c}
\sin \omega \tau^{\prime} \\
\cos \omega \tau^{\prime}
\end{array}\right) f\left(t-\tau^{\prime}\right) \\
& =\frac{f(t)}{\omega}\left(\begin{array}{l}
1 \\
0
\end{array}\right)-\frac{1}{\omega} \int_{0}^{t} \mathrm{~d} \tau\left(\begin{array}{c}
-\cos \omega \tau^{\prime} \\
\sin \omega \tau^{\prime}
\end{array}\right) \frac{d}{d \tau} f(t-\tau) .
\end{aligned}
$$

The second term on the last r.h.s. of Eq. (8) can be made arbitrarily small by making the switching-on process arbitrarily slow. The first term corresponds to Eq. (7), because $f(t)=f_{0}$ after the transient switching on regime is over. It is thus clear that the stationary solution, Eq. (7), can be described as an interference of contributions created by ultrashort impulses.

When an ensemble of $N$ classical systems is treated, we might be interested in an ensemble value of some quantity, e.g.

$$
\Phi(t)=\frac{1}{N} \sum_{n} \phi_{n}(t)=\langle\phi(t)\rangle .
$$

Here $\phi_{n}(t)$ is the phase vector of an individual member of the ensemble, which might be in principle assigned its own Green's function $G_{n}(t)$ and initial condition $\varphi_{0}^{(n)}$. If we assume these to be identical for all members of the ensemble, the average in Eq. (9) can be replaced by $\phi(t)$ itself, i.e. $\Phi(t)=\phi(t)$.

One can reformulate the result, Eq. (6), in terms of a formal virtual ensemble. One can assume that all members of the ensemble are identical, but the constant driving is in fact not constant for each individual member of the ensemble, rather, it is constant only in its effect of the ensemble quantity, so that

$$
\Phi(t)=\sum_{n} \int_{0}^{\infty} \mathrm{d} \tau G(t-\tau) \varphi_{0} f_{n}(\tau)
$$

with

$$
f(t)=\sum_{n} f_{n}(t)
$$

where $f_{n}(t)$ acts only on the $n^{t h}$ system of the ensemble. We can further assume that the force $f_{n}(t)$ acts only during a very short interval $\Delta t$ after time $t_{n}$, where the times $t_{n}$ are distributed on the time axis with the step $\Delta t$. The $f_{n}(t)$ then reads as

$$
f_{n}(t)=N f(t) \Theta\left(t-t_{n}\right) \Theta\left(-\left(t-\left(t_{n}+\Delta t\right)\right)\right),
$$

where the two Heaviside step functions $\Theta(t)$ ensure that the function $f_{n}(t)$ is equal to $f(t)$ on the interval $\left[t_{n} ; t_{n}+\right.$ $\Delta t]$ and zero otherwise. When $\Delta t$ is infinitesimal, Eq. (11) can be written as

$$
f(t)=\sum_{n} \Delta t f\left(t_{n}\right) \frac{\Theta\left(t-t_{n}\right) \Theta\left(-\left(t-\left(t_{n}+\Delta t\right)\right)\right)}{\Delta t},
$$

where we replaced $f(t)$ by its values $f\left(t_{n}\right)$ at the beginning of the interval $\left[t_{n} ; t_{n}+\Delta t\right]$. The sum over $n$ is meant here as a sum over times at which these molecules are being driven by the force $f_{n}(t)$. In the limit of $\Delta t \rightarrow 0$, we can write Eq. (13) as an integral

$$
f(t)=\int_{0}^{\infty} \mathrm{d} t^{\prime} f\left(t^{\prime}\right) \delta\left(t-t^{\prime}\right) .
$$


The integral over $t^{\prime}$ could still be understood as an integral over an ensemble of molecules. The overall vector for the ensemble is therefore:

$$
\begin{aligned}
\Phi(t) & =\int_{0}^{\infty} \mathrm{d} t^{\prime} \int_{0}^{\infty} \mathrm{d} \tau G(t-\tau) \varphi_{0} \delta\left(\tau-t^{\prime}\right) f\left(t^{\prime}\right) \\
& =\int_{0}^{\infty} \mathrm{d} t^{\prime} G\left(t-t^{\prime}\right) \varphi_{0} f\left(t^{\prime}\right)=\phi(t) .
\end{aligned}
$$

The first line of the last equation should be interpreted as a sum of dynamics of individual systems which have been excited at respective times $t-t^{\prime}$ in the past and oscillate without relaxation ever since. The second line highlights the fact that this is equivalent to the general solution for a single system $\phi(t)$ (cf. Eq. 6).

The equations of motion of a classical driven oscillator represent therefore at least two very different physical problems: either (i) a single oscillator driven by a continuous force or (ii) an ensemble of oscillators driven each by an impulsive force. Because the resulting dynamics is the same, we can always represent single oscillator by a virtual ensemble of oscillators, whenever it is advantageous in the mathematical treatment of the problem.

Classical dynamics with an instantaneous excitation is only a mathematical prerequisite for the calculation of an actual dynamics of the system, which is determined by the driving force. On the other hand, one can imagine a physical representation of the action of a steady force $f(t)$ and its decomposition Eq. (11), say, by a stream of particles with small cross-section acting on an ensemble of oscillators. Each collision leads to a transition of momentum from the particle to the oscillator, and the oscillator follows Eq. (3) with an instantaneous driving force. The average quantity $\Phi(t)$ follows Eq. (10) or equivalently Eq. (15). This physical representation of Eq. (15) seems to form a basis of an intuitive picture of quantized light acting on an ensemble of molecules. While in the above classical picture it is quite natural to deal with instantaneous excitation events, we will see in the following sections that quantum mechanics prevents us from introducing such ultrafast excitations. The instantaneous quantum jumps seem to be only a simplified interpretation imported to quantum mechanics from classical mechanics.

\section{EXCITATION OF AN OPEN QUANTUM SYSTEM BY LIGHT}

In this section we will deal with the problem of external driving from a quantum mechanical perspective. Let us introduce the Hamiltonian $H$ of a system consisting in a molecular aggregate $M$ interacting with a radiation $R$, its immediate environment (or bath) $B$ and their mutual interactions

$$
H=H_{R}+H_{B}+H_{M}+H_{M-B}+H_{M-R} .
$$

Here, $H_{M-B}$ is the interaction between the molecular system (or more precisely its selected DOF, such as electronic states) with the environment, and $H_{M-R}$ is the interaction of the molecular system with light. We neglect the interaction of the environment with the light and assume that the interaction of the molecular subsystem with the light is weak.

The time evolution of the system is given by the Schrödinger equation $i \hbar \frac{\partial}{\partial t}|\psi(t)\rangle=H|\psi(t)\rangle$. Typically, the molecular system is in its electronic ground state $|g\rangle$ before the light is applied. It is excited by light into a band of states $|n\rangle$. We define complementary projectors $P_{g}=|g\rangle\langle g|$ and $P_{e}=\sum_{n}|n\rangle\langle n|$ which define the unity operator $1 \equiv P_{g}+P_{e}$ on the relevant electronic Hilbert space. We ignore all dark states or states that could be reached by multiple excitation by light (such as two-exciton states etc.) because we assume weak lightmatter interaction which allows for a linearization in the interaction Hamiltonian $H_{M-R}$.

Motivated by the usual situation in photosynthetic antennae, we assume that the molecular Hamiltonian $H_{M}$ does not include terms directly connecting the ground state with the excited states (the transitions between electronic states separated by optical gap are only mediated by light). Similarly, the system-bath interaction $H_{M-B}$ does not cause such transitions on the time-scales similar or shorter than the excitation energy transfer. These properties are expressed in the relations

$$
P_{g} H_{M} P_{e}=P_{g} H_{M-B} P_{e}=0 .
$$

The system-light interaction term, on the other hand, contains just terms causing transitions between the ground- and excited states and therefore

$$
P_{e} H_{M-R} P_{e}=0
$$

The operators $H_{R}$ and $H_{B}$ do not depend on the electronic DOF of the molecular subsystem and thus $P_{e} H_{R} P_{e}=P_{g} H_{R} P_{g}=H_{R}$, and similarly for $H_{B}$. We will define the system-bath interaction Hamiltonian so that $P_{g} H_{M-B} P_{g}=0$ as any difference from zero in $H_{M-B}$ of the ground state can be added to the Hamiltonian $H_{B}$. With the following definitions

$$
\begin{gathered}
H_{e} \equiv P_{e} H_{M} P_{e}, H_{g} \equiv P_{g} H_{M} P_{g} \\
H_{e g} \equiv P_{e} H_{M-R} H_{g}, \Delta V \equiv P_{e} H_{M-B} P_{e} \\
\left|\psi_{g}(t)\right\rangle \equiv P_{g}|\psi(t)\rangle,\left|\psi_{e}(t)\right\rangle \equiv P_{e}|\psi(t)\rangle
\end{gathered}
$$

we can write two coupled Schrödinger equations for the ground state and the excited state bands

$$
\begin{aligned}
i \hbar \frac{\partial}{\partial t}\left|\psi_{e}(t)\right\rangle= & \left(H_{R}+H_{B}+H_{e}+\Delta V\right)\left|\psi_{e}(t)\right\rangle \\
& +H_{e g}\left|\psi_{g}(t)\right\rangle
\end{aligned}
$$




$$
\begin{aligned}
i \hbar \frac{\partial}{\partial t}\left|\psi_{g}(t)\right\rangle & =\left(H_{R}+H_{B}+H_{g}\right)\left|\psi_{g}(t)\right\rangle \\
& +H_{g e}\left|\psi_{e}(t)\right\rangle .
\end{aligned}
$$

The last term in Eq. (23) will be neglected as we aim for an equation for excited state evolution which is linear in $H_{M-R}$. We also set the electronic energy of the ground state to zero, i.e. $H_{g}=0$. Given an initial condition $|g\rangle$ at time $t=0$, we can solve Eq. (23), obtaining

$$
\left|\psi_{g}(t)\right\rangle=U_{R}(t) U_{B}(t)|g\rangle,
$$

where we have defined the evolution operators $U_{R}(t)=$ $\exp \left\{-i H_{R} t / \hbar\right\}$ and $U_{B}(t)=\exp \left\{-i H_{B} t / \hbar\right\}$.

The solution of Eq. (22) can now be found by using the solution for the ground state, Eq. (24), and finding the Green's function of its homogenous part

$$
G(t)=\Theta(t) U_{R}(t) \tilde{G}(t) U_{B}(t),
$$

where we defined

$$
\tilde{G}(t)=\exp _{\leftarrow}\left\{-\frac{i}{\hbar}\left(H_{e} t+\int_{0}^{t} \mathrm{~d} \tau \Delta V(-\tau)\right)\right\},
$$

$\Delta V(t)=U_{B}^{\dagger}(t) \Delta V U_{B}(t)$ and $H_{e g}(t)=U_{R}^{\dagger}(t) H_{e g} U_{R}(t)$. We obtain

$$
\left|\psi_{e}(t)\right\rangle=U_{R}(t) \int_{0}^{t} \mathrm{~d} \tau \tilde{G}(t-\tau) H_{e g}(t) U_{B}(t)|g\rangle .
$$

From Eq. (27), we can easily construct the density operator describing the excited state part of the total system

$$
\hat{W}(t)=\left|\psi_{e}(t)\right\rangle\left\langle\psi_{e}(t)\right| .
$$

The corresponding reduced density matrix (RDM) of the molecular system is obtained by averaging quantum mechanically over the radiational and the environmental DOF: $\hat{\rho}(t)=\operatorname{Tr}_{R}\left[\operatorname{Tr}_{B}\{\hat{W}(t)\}\right]$. The system-light interaction Hamiltonian will be assumed in dipole approximation

$$
H_{e g}(t)=-\hat{\mu}_{e g} \hat{\mathcal{E}}^{(+)}(t),
$$

where $\hat{\mu}_{e g}=P_{e} \hat{\mu} P_{g}$ is a projection of the transition dipole moment operator, and $\hat{\mathcal{E}}^{(+)}$is the component of the electric field operator containing the field annihilation operator. The RDM can now be written as

$$
\hat{\rho}(t)=-\int_{0}^{t} \mathrm{~d} \tau \int_{0}^{t} \mathrm{~d} \tau^{\prime} R_{\text {light }}\left(t, \tau, \tau^{\prime}\right) I_{\text {light }}\left(\tau, \tau^{\prime}\right),
$$

with

$$
\begin{array}{r}
R_{\text {light }}\left(t, \tau, \tau^{\prime}\right)=\left(\frac{i}{\hbar}\right)^{2} \operatorname{Tr}_{B}\left\{\tilde{G}(t-\tau) \hat{\mu}_{e g}\right. \\
\left.\times U_{B}(t)\left|\phi_{g}\right\rangle\left\langle\phi_{g}\right| U_{B}^{\dagger}(t) \hat{\mu}_{g e} \tilde{G}^{\dagger}\left(t-\tau^{\prime}\right)\right\},
\end{array}
$$

$$
I_{\text {light }}\left(\tau^{\prime}, \tau\right)=\operatorname{tr}_{R}\left\{\hat{\mathcal{E}}^{(-)}\left(\tau^{\prime}\right) \hat{\mathcal{E}}^{(+)}(\tau)|\Xi\rangle\langle\Xi|\right\} .
$$

This is a convenient version of the result from Ref. [44] where we assumed $\left|\psi_{0}\right\rangle=|g\rangle\left|\varphi_{g}\right\rangle|\Xi\rangle$, with $|\Xi\rangle$ representing the state of the light at $t=0$ and $\left|\varphi_{g}\right\rangle$ representing the state of the environment. The operator $R_{\text {light }}\left(t, \tau, \tau^{\prime}\right)$ describes the molecular system response to the action of weak light, which is described by the field correlation function $I_{\text {light }}\left(\tau, \tau^{\prime}\right)$.

Considering quantized light and the Bose-Einstein distribution of the modes populations $\bar{n}(\omega)=1 /\left(e^{\beta \hbar \omega}-1\right)$, we can arrive at a convenient expression for the correlation function of light

$$
I\left(\tau^{\prime}, \tau\right)=\int_{0}^{\infty} d \omega \frac{\hbar}{\varepsilon_{0} 2 \pi^{2} c^{3}} \frac{\omega^{3}}{e^{\beta \hbar \omega}-1} e^{i \omega\left(\tau^{\prime}-\tau\right)},
$$

where the integrated expression without the exponential is the Planck's law, denoted $W(\omega)$ in the following. We thus obtain that the correlation function is stationary, i.e. only function of the difference $\left(\tau-\tau^{\prime}\right)$ and it can be expressed as a Fourier transform of the spectrum of the light,

$$
\mathcal{G}(\tau) \equiv I\left(\tau^{\prime}, \tau\right)=\int_{0}^{\infty} d \omega W(\omega) e^{i \omega \tau} .
$$

This formula holds generally for stationary light and is called Wiener-Khintchine theorem [48]. Note the importance of Eq. (34) which gives the possibility to estimate the light coherence time directly from its spectrum $W(\omega)$. Also, this allows us the write Eq. (30) in a form

$$
\begin{aligned}
\hat{\rho}(t)= & -\int_{0}^{\infty} \mathrm{d} \omega W(\omega) \\
& \times\left(\int_{0}^{t} \mathrm{~d} \tau \int_{0}^{\tau} \mathrm{d} \tau^{\prime} R_{\text {light }}\left(t, \tau, \tau^{\prime}\right) e^{i \omega\left(\tau^{\prime}-\tau\right)}+\text { h.c. }\right),
\end{aligned}
$$

using the fact that $R_{\text {light }}\left(t, \tau, \tau^{\prime}\right)$ is a Hermitian operator and integrating separately the parts where $\tau>\tau^{\prime}$ and $\tau<\tau^{\prime}$, respectively. The abbreviation h.c. represents the Hermitian conjugated term. In the case that the driven system consists of a single excited state $|a\rangle$, the response takes a form

$$
\begin{aligned}
\rho_{a a}(t) & =\frac{\left|\left\langle a\left|\hat{\mu}_{e g}\right| g\right\rangle\right|^{2}}{\hbar^{2}} \\
& \times 2 \operatorname{Re} \int_{0}^{\infty} \mathrm{d} \omega W(\omega) \int_{0}^{t} \mathrm{~d} \tau \int_{0}^{\tau} \mathrm{d} \tau^{\prime} \mathrm{e}^{\mathrm{i} \omega \tau^{\prime}} \mathrm{e}^{-\mathrm{g}\left(\tau^{\prime}\right)-\mathrm{i} \omega_{\mathrm{ag}} \tau^{\prime}},
\end{aligned}
$$

where we can identify the absorption spectrum

$$
\alpha(\omega) \approx\left|\left\langle a\left|\hat{\mu}_{e g}\right| g\right\rangle\right|^{2} 2 \operatorname{Re} \int_{0}^{\infty} \mathrm{d} \tau e^{i \omega \tau} e^{-g(\tau)-i \omega_{a g} \tau},
$$


and $g(t)$ the so-called line shape function (Eq. (8.13) in [47]). For times $t$ long relative to the decay time of the linear response, i.e. after $e^{-g(t)} \approx 0$, we get

$$
\rho_{a a}(t) \approx \int \mathrm{d} \omega W(\omega) \alpha(\omega)
$$

The population is thus proportional to the overlap of the light-spectrum and the absorption spectrum of the molecular system, which is an expected result.

\section{EXCITATION BY NEIGHBORING ANTENNA}

Let us now briefly show that Eq. (30) describes also another important situation in photosynthesis, namely, the situation when the relevant molecular system is excited by a neighboring antenna. For this, we only have to replace the light radiation by an antenna $-(R \rightarrow A)$ in Eq. (16). More specifically, we replace the Hamiltonian $H_{R}$ of the radiation by the Hamiltonian of the neighboring antenna

$$
H_{A}=H_{B_{A}}+\sum_{k_{A}, l_{A}} H_{k_{A} l_{A}}\left|k_{A}\right\rangle\left\langle l_{A}\right|,
$$

where $\left|k_{A}\right\rangle$ and $\left|l_{A}\right\rangle$ are the electronic excited states of the neighboring antenna, $B_{A}$ denotes the environment of the antenna, $H_{B_{A}}$ is its associated Hamiltonian and $H_{k_{A} l_{A}}$ describes the interaction of the antenna electronic states with its environment. These can also cause transitions between excited states. The details of the Hamiltonian $H_{A}$ are not of crucial importance here. The role of the system-light interaction operator is now taken by the resonance interaction Hamiltonian

$$
H_{M-A}=\sum_{k_{A}}\left(\sum_{n} J_{n k_{A}}|n\rangle\langle g|\right)\left(\left|g_{A}\right\rangle\left\langle k_{A}\right|\right)+\text { h.c.. }
$$

Here, $J_{n k_{A}}$ is the resonant interaction energy between the state $\left|k_{A}\right\rangle$ of the neighboring antenna and the state $|n\rangle$ of the molecular system of interest. The Hamiltonian $H_{M-A}$ has a somewhat more complicated structure than the light-matter interaction Hamiltonian and it cannot be in general written as a product of two operators, each belonging to the Hilbert space of one of the interacting entities. This complicates the matter only slightly. For simplicity, let us assume that the excitation is transferred only to one state $|m\rangle$ of the molecular system. Then the field operator $\hat{\mathcal{E}}^{(+)}$in Eq. (32) will be replaced with the operator $\hat{\mathcal{A}}^{(+)}=\sum_{k_{A}} J_{m k_{A}}\left|g_{A}\right\rangle\left\langle k_{A}\right|$ and the operator $\hat{\mu}_{e g}$ simply by $\hat{p}_{e g}=|m\rangle\langle g|$. We obtain an operator for the molecular response $R_{\text {antenna }}\left(t, \tau, \tau^{\prime}\right)$ in which $\hat{\mu}_{e g}$ is replaced by $\hat{p}_{e g}$ and instead of the light correlation function we have an antenna energy gap correlation function

$I_{\text {antenna }}\left(\tau, \tau^{\prime}\right)=\operatorname{Tr}_{B_{A}}\left\{\hat{\mathcal{A}}^{(-)}\left(\tau^{\prime}\right) \hat{\mathcal{A}}^{(+)}(\tau)\left|\phi_{A}(0)\right\rangle\left\langle\phi_{A}(0)\right|\right\}$
The state $\left|\phi_{A}(0)\right\rangle=\left|\phi_{M_{A}}(0)\right\rangle\left|\phi_{B_{A}}(0)\right\rangle$ represents the initial state of the antenna (composed of a molecular aggregate and a bath) at $t=0$, and the trace is taken over the antenna environment.

If in addition, we assume the neighboring antenna to be represented by a single level $|l\rangle \equiv\left|l_{A}\right\rangle$, it is easy to show that the expression Eq. (30) with $I_{\text {antenna }}$ and $R_{\text {antenna }}$ represents a Förster type energy transfer. Under these approximations, the response reads

$R_{\text {antenna }}\left(t, \tau, \tau^{\prime}\right)=\frac{1}{\hbar^{2}} \operatorname{Tr}_{B}\left\{\tilde{G}_{m}\left(\tau^{\prime}-\tau\right) \hat{W}_{B}\right\}=\frac{1}{\hbar^{2}} e^{-g\left(\tau^{\prime}-\tau\right)}$

where we evaluated the trace over the bath by second cumulant expansion [49]. The antenna correlation function reads

$$
\begin{aligned}
I_{\text {antenna }}\left(\tau, \tau^{\prime}\right) & =\left|J_{l m}\right|^{2} \operatorname{Tr}_{B_{A}}\left\{U_{A}^{\dagger}\left(\tau^{\prime}-\tau\right)|l\rangle\left\langle g_{A}\right|\right. \\
& \left.\times U_{A}\left(\tau^{\prime}-\tau\right)\left|g_{A}\right\rangle\langle l| \hat{W}_{B_{A}}\right\} \rho_{l l}^{(A)}(0),
\end{aligned}
$$

where $\rho_{l l}^{(A)}(0)=\operatorname{Tr}_{B_{A}}\left\{\left|\phi_{M_{A}}(0)\right\rangle\left\langle\phi_{M_{A}}(0)\right|\right\}$ is the population of the level $|l\rangle, \hat{W}_{B_{A}}=\left|\phi_{B_{A}}(0)\right\rangle\left\langle\phi_{B_{A}}(0)\right|$ is the density operator of the antenna environment at initial time, and $U_{A}(t)=\exp \left\{-\frac{i}{\hbar} H_{A} t\right\}$ is the evolution operator with the Hamiltonian of the antenna. Because we limited the relevant states of the antenna on a single state $|l\rangle$ and assumed that it is initially excited, i.e. $\left|\phi_{M_{A}}(0)\right\rangle=|l\rangle \Leftrightarrow \rho_{l l}^{(A)}(0)=1$, Eq. (43) can also be evaluated by cumulant expansion - note that the evaluation needs a special care because the antenna is initially in the excited state. Similar problem is solved when describing spectrum of spontaneous emission (see e.g. Chap. 8 in [47]) and the solution reads

$$
I_{\text {antenna }}\left(\tau, \tau^{\prime}\right)=\left|J_{l m}\right|^{2} e^{-g_{A}^{*}\left(\tau^{\prime}-\tau\right)-2 i \lambda_{A}\left(\tau^{\prime}-\tau\right)+i \omega_{l m}\left(\tau^{\prime}-\tau\right)} .
$$

In case of a single acceptor and a single donor states, Eq. (30) thus yields

$$
\begin{aligned}
& \rho_{m m}(t)=\frac{2\left|J_{l m}\right|^{2}}{\hbar^{2}} \\
& \times \operatorname{Re} \int_{0}^{t} \mathrm{~d} \tau \int_{0}^{\tau} \mathrm{d} \tau^{\prime} e^{-g(\tau)-g_{A}^{*}(\tau)-2 i \lambda_{A} \tau-i \omega_{m l} \tau} \rho_{l l}^{(A)}(0),
\end{aligned}
$$

where we split the double integral from 0 to $t$ into parts with $\tau>\tau^{\prime}$ and $\tau<\tau^{\prime}$ and used the property of the line shape function $g(-t)=g^{*}(t)$. Taking a time derivative of Eq. (45) we obtain a rate equation with a time dependent rate

$$
K_{m l}(t)=\frac{2\left|J_{l m}\right|^{2}}{\hbar^{2}} \operatorname{Re} \int_{0}^{t} \mathrm{~d} \tau e^{-g(\tau)-g_{A}^{*}(\tau)-2 i \lambda_{A} \tau-i \omega_{m l} \tau}
$$

For times $t$ when the integrand of Eq. (46) decays to zero, we can replace the upper limit of the integral by 
$\infty$. We have discussed the relation between the correlation function of the light and its spectrum in the previous section. In the same general way we can show that the rate given by Eq. (46), for $t \rightarrow \infty$, has a form of the overlap of the absorption spectrum of the excitation acceptor and the emission spectrum of the donor. We have thus obtained an expression which reduces to the standard Förster resonant energy rate. For a general case of energy transfer between two multilevel systems, Eq. (30) is equivalent to the corresponding generalized Förster rate description. This demonstrates the validity and generality of Eq. (30) for weakly interacting systems. Eq. (30) therefore provides us with a general platform for discussing the possibility of excitation of dynamic coherence under different conditions and studying the relevance of dynamic coherence for the dynamics in excited state.

One might raise a question about the validity of Eq. (30) for the driving by light or neighboring antenna lasting for times during which the probability of capturing more than one photon per molecule becomes significant. Obviously, if the driven system were limited to some small antenna and the excitation remained on this antenna for long times, the usual single exciton manifold limitation of the theoretical description would represent a serious drawback. However, when the driven system is sufficiently large (e.g. containing other antennae as well as the reaction center and the different chemical states of the photosynthetic apparatus into which the excitation is transferred during the photosynthetic process) the probability of finding two excitations on a particular antenna remains very low during the whole duration of illumination. The proper description of an antenna in operation is a steady state description, where all populations vary only slowly (see e.g. Ref. [50]). We should emphasize that expressions such as Eqs. (38) and (45) only represent an illustrative example but that the expression given in Eq. (30) can be applied to large system and is not even limited to just the excitonic part of the photosynthetic apparatus.

\section{TIME-SCALE OF EXCITATION EVENTS}

It was argued in Refs. [42 44, 51] that the state $\hat{\rho}(t)$ produced by Sun light (or in fact by any stationary source) does not contain any dynamic coherences, and that the dynamic coherence is therefore irrelevant for the natural photosynthetic processes. Dynamic coherence can only be created by excitation of sufficiently short duration. The counter argument against this conclusion is often based on the short correlation time of the Sun light, and on a picture where Eq. (30) represents an ensemble of molecules in which each molecule has a specific time of photon capture. This is the bullet-like picture of the photon. The state, Eq. (30), is then viewed as a statistical average over molecules individually excited at specific times. In the following we will show that the bullet-like picture of the photon is not consistent with the finite correlation time of Sun light. It is not possible to write the quantum mechanical result, Eq. (30), in terms of sudden excitations in the case that both the correlation function of light or the material response has a finite correlation time. While such a formal decomposition into sudden excitations was possible for classical systems, in quantum mechanics it is not possible, not even formally. Identifying the dependence of the relevant time-scale of the excitation process on the light spectrum, we suggest a simple experiment to test the relevance of this time scale for the yield of photosythesis.

The dynamics of the state vector in Eq. (27) can certainly be understood as an integral over instantaneous jumps in the state vector of the molecular system. However, the result of an experiment is described by an expectation value

$$
\langle\hat{A}\rangle \equiv\langle\psi(t)|\hat{A}| \psi(t)\rangle=\operatorname{tr}\{\hat{A}|\psi(t)\rangle\langle\psi(t)|\}
$$

which is quadratic in the state vector. Two interactions with the external fields (one from the bra and one from the ket side of the expression) are needed to produce the excited state population. In general, these excitations can occur at different times. Let us select two infinitesimal contributions to the excited state part $\left\langle n \mid \psi_{e}(t)\right\rangle$ of the wavefunction from Eq. (27), where $|n\rangle$ is a selected state from the excited state band. For simplicity we assume that during the time interval of interest, the state $|n\rangle$ does not relax to other states. Let us assume that the excitations occured at times $t_{1}$ and $t_{2}$, with $t_{2}>t_{1}$. At time $t_{1}$ the state of the system corresponds to $\left|\psi_{g}\left(t_{1}\right)\right\rangle=U_{B}\left(t_{1}\right) U_{R}\left(t_{1}\right)|g\rangle$ (see Eq. (24) ) and an infinitesimal contribution to the excitated state in Eq. (27) reads

$$
\left|\psi_{e}^{\left(t_{1}\right)}\left(t_{1}\right)\right\rangle=-\frac{i}{\hbar} \hat{\mu}_{e g} \hat{\mathcal{E}}^{(+)}\left|\psi_{g}\left(t_{1}\right)\right\rangle \mathrm{d} t .
$$

An infinitesimal contribution excited at time $t_{2}$ reads

$$
\left|\psi_{e}^{\left(t_{2}\right)}\left(t_{2}\right)\right\rangle=-\frac{i}{\hbar} \hat{\mu}_{e g} \hat{\mathcal{E}}^{(+)}\left|\psi_{g}\left(t_{2}\right)\right\rangle \mathrm{d} t .
$$

At time $t_{2}$, however, the contribution excited at $t_{1}$ has already evolved for time $\left(t_{2}-t_{1}\right)$ according to the excited state Hamiltonian $H_{e}$, that is by evolution opertor $U_{e}\left(t_{2}-t_{1}\right)=\exp \left\{-i H_{e}\left(t_{2}-t_{1}\right) / \hbar\right\}$. It reads $\left|\psi^{\left(t_{1}\right)}\left(t_{2}\right)\right\rangle=U_{e}\left(t_{2}-t_{1}\right)\left|\psi^{\left(t_{1}\right)}\left(t_{1}\right)\right\rangle$. The contribution to the population of some excited state $|n\rangle$ at time $t_{2}$ (and times $t>t_{2}$ ) is obtained by combining these two infinitesimal contributions

$$
\delta p_{n}\left(t_{2}, t_{1}\right)=2 \operatorname{Re}\left\langle\psi^{\left(t_{2}\right)}\left(t_{2}\right) \mid n\right\rangle\left\langle n \mid \psi^{\left(t_{1}\right)}\left(t_{2}\right)\right\rangle .
$$

The real part is a result of the fact that in Eq. (27) we integrate symmetrically along the times $\tau>\tau^{\prime}$ and times $\tau<\tau^{\prime}$. Eq. (50) leads to

$$
\delta p_{n}\left(t_{2}, t_{1}\right)=\frac{2\left|\mu_{e g}\right|^{2}}{\hbar^{2}}(\mathrm{~d} t)^{2}
$$




$$
\times \operatorname{Re}\left\{e^{-g_{n n}\left(t_{2}-t_{1}\right)-i \omega_{n g}\left(t_{2}-t_{1}\right)} I\left(t_{2}, t_{1}\right)\right\},
$$

where we used cumulant expansion to evaluate the molecular response, and we used the definition of the light correlation function, Eq. (32). For stationary light, the correlation function depends only on the difference $t_{2}-t_{1}$. Two excitations by the same field occuring in the wavefunction at times separated by a delay longer than the life-time of the optical coherence created in the material or the coherence time of light do not contribute together to the population. For light with long coherence time, detectors with sufficiently fast response, i.e. high time resolution, can detect individual excitation events, i.e. photon arrivals, because different outcomes of the detection are separated from the particular measured one by decoherence. This explains the presence of individual "clicks" of a detector for the light with coherence time longer than the time resolution of the detector. The posibility of a faster than coherence time resolution of the detector, however, does not mean that prior to the measurement, the light is already in a state where arrival times are decided. On the other hand, it is important to stress that Sun light has a spectrum broader than the absorption spectra of photosynthetic systems, and therefore the correlation time of Sun light is shorter than the optical dephasing time of the antennae. The characteristic length of the excitation event in photosynthesis is therefore indeed decided by the light.

The correlation function of the Sun light has nevertheless a finite characteristic coherence time which is given, according to Eq. (34), by the Fourier transform of its spectrum. The statistical description of a quantum mechanical system based on instantaneous quantum jumps in the wavefunction is not appropriate for systems with finite response time or light with finite coherence time. It is interesting that such a statistical description is possible for linear classical systems.

While a decomposition of the light action on a molecular system in terms of instantaneous jumps is not possible, nothing can prevent us from following the suggestion of Ref. [41] were it was suggested to represent the Sunlight fluctuations by a series of coherent spikes of short duration occurring at some times $t_{k}$, so that

$$
I\left(\tau, \tau^{\prime}\right)=\sum_{k} I_{k}\left(\tau, \tau^{\prime} ; t_{k}\right)=\sum_{k} \mathcal{G}_{k}\left(\tau-\tau^{\prime} ; t_{k}\right) .
$$

Obviously, if the correlation function is only rewritten in the new way and each molecule feels the same set of spikes, then the predictions of Refs. [43, 44] are valid for individual molecules and no coherence can be generated even on a single molecular level. This argument is similar to the one put forward in Ref. [17] because here it also does not alter what is the order of averaging in Eq. (47). In order for the proposal of Ref. [41] to be non-trivial we would have to accept an interpretation of Eq. (52), in which each molecule of the ensemble feels e.g. just one component of the decomposition. Correspondingly, each molecule would be subject to some microscopic field state
$\left|\Xi_{k}\right\rangle$ for which $I_{k}\left(\tau, \tau^{\prime} ; t_{k}\right)=\operatorname{Tr}_{R}\left\{\hat{\mathcal{E}}(\tau) \hat{\mathcal{E}}\left(\tau^{\prime}\right)\left|\Xi_{k}\right\rangle\left\langle\Xi_{k}\right|\right\}$. The fact that we detect a macroscopic correlation function $I\left(\tau, \tau^{\prime}\right)$ would then be a result of the fact that our detectors are macroscopic. The expansion Eq. (52) could in principle enable us to simulate the finite time scale of the excitation events. Here we would no more be exciting all molecules by the same averaged external field, and correspondingly, if the short excitation time aspect of the excitation process has an influence on the yield of the photosynthetic process, the sum of contributions of the individual molecules would not be guaranteed to yield the same result as in the case where all molecules are excited by one averaged field. This case is however beyond the validity of Eq. (30) in which the decomposion of Eq. (52) does not affect the final macroscopic state of the system in any way and correspondingly it has no influence on the yield.

Interestingly, one can relatively easily test the proposition of Ref. [41] experimentally, without any use of a complicated time-resolved spectroscopy apparatus. For this experimental test one has to be able to measure the macroscopic yield of the light capture and the transfer throught a given antenna, e.g. in terms of the number of excitations transferred to the RC. The measurement can be performed with the Sun light (carefully attenuated in order to prevent the self regulating mechanisms of photosynthesis to decrease the optimal yield). This is a situation where Eq. (34) suggests that individual excitations occur on a short time scale. Then one can repeat the same measurement with a monochromator, selecting spectrally narrow parts of the Sun-light spectrum. Eq. (34) predicts that in this case, the time-scale of the interaction with light is much longer. When the spectrum of the light is narrower than the spectral distance between electronic levels of the antenna, the time-scale of the excitation allowed by the light spectrum is already too long to create any coherences between the two electronic levels. Finally, one can integrate the yield across the whole Sun spectrum and compare with the total yield achieved by the total Sun spectrum.

Eq. (30) predicts that the yields are the same. Notice that it is easy to construct a case where the yields were not the same. If, for instance, the photosynthetic process to be completed required first an excitation at certain wavelength followed by an excitation at another wavelegth, then a single spectral component would reasult in no yield. This has however nothing to do with the coherent excitation, and this case is in fact beyond the validity of Eq. (30) which assumes that we always start with exciting the ground state of the antenna. Extending the present formalism to more complicated initial state of excitation is however possible. 


\section{DYNAMIC COHERENCE AND DELOCALIZATION UNDER VARIOUS EXCITATION CONDITIONS}

The experiment suggested in the previous section could in principle decide the question raised by the detection of long lived coherence in photosynthetic antenna. On the other hand, anticipating its result, we have every reason to believe that the quantum mechanical description represented by Eq. (30) is valid and the coherence could therefore have an influence on the expectation values relevant for the yield of photosynthesis only if it showed in the averaged density matrix. The conclusion of Refs. [42 44, 51] is that Sun light does not produce any dynamic coherence in the averaged state of a driven photosynthetic antenna. It is less obvious how much coherence is created when excitation is transferred into a molecular system from a neighboring molecular system and this question will be examined in this section. As a model for this case we take here the chlorosome-FMO complex of the Chlorobium tepidum bacteria, with the chlorosome simplified into a single pigment of its baseplate. The FMO complex [52] is a homotrimer consisting of bacteriochlorophyll-a (BChl-a) molecules that transfer excitation energy between the baseplate protein of the chlorosome and the reaction center. We study the EET dynamics considering each one of the monomers to be independent and composed of seven BChl-a (system Hamiltonian from Ref. 53]). According to the recent crystallographic studies reporting the existence of an eighth BChl molecule [54, 55], we studied the holo form (eight BChls per monomer, Hamiltonian from Ref. [56]), which presented a similar dynamics (therefore not presented here). Based on theoretical calculations [53] and recent experimental verification [57], the FMO complex is assumed to be oriented with BChls 1 and 6 toward the base plate protein, whereas BChls 3 and 4 are at the interface between the FMO and the reaction center. Accordingly, the initial excited pigments in our numerical calculations are chosen to be either BChls 1 or 6 .

For our purpose, we postulate a simple time independent variant of Eq. (46) with a single transfer rate from the chlorosome baseplate to the FMO and constant rates for the internal energy relaxation inside the FMO. For the purpose of demonstration we want to describe correctly the relevant time-scales of the FMO's coherent oscillations, i.e. we will use the Hamiltonian available in literature, [53]. We describe energy transfer by secular Redfield tensor calculated assuming Debye spectral density (see e.g. Eq. (3.268) in Ref. [58]) with a cut-off frequency $\omega_{D}=100 \mathrm{~cm}^{-1}$ and reorganization energy $\lambda=$ $35 \mathrm{~cm}^{-1}$ (as in Ref. [59]) to describe the energy gap fluctuations on the FMO pigments. These parameters give us relaxation and dephasing dynamics time-scales similar to previous theoretical works.

In order to quantify the coherence in the system, we define a measure of the dynamic coherence $\Xi$, as:

$$
\Xi=\frac{\sum_{\alpha>\beta} \xi_{\alpha \beta} \cdot\left(\rho_{\alpha \alpha}+\rho_{\beta \beta}\right)}{(N-1) \sum_{1}^{N} \rho_{\alpha \alpha}} .
$$

Here $\xi_{\alpha \beta}=\frac{\left|\rho_{\alpha \beta}\right|^{2}}{\rho_{\alpha \alpha} \rho_{\beta \beta}}$ defines the relative amplitude of the coherences between the two excited states $\alpha$ and $\beta$ ( $\xi=1$ for a completely coherent system, $\left.\rho_{\alpha \beta}=\langle\alpha|\rho| \beta\rangle\right)$. This quantity is weighted in Eq. (53) by the population probabilities of the corresponding states to only account for the significantly populated states. It is easy to verify that $\Xi=1$ for a purely coherent system and $\Xi=0$ when no superposition is present. Note that the amount of coherence is a time-dependent variable when the system of interest is subjected to relaxation due to some interaction with bath DOF. It is a basis dependent quantity in line with the basis-dependent properties of the dynamic coherences. Unlike established measures such as the purity, the quantity $\Xi$, when expressed in the eigenstate basis, is directly representative of the dynamic coherences as experimentally accessible in the non-linear spectroscopy. When expressed in the basis of states local to the molecules forming the aggregate, the quantity $\Xi$ can also quantify the delocalization of a given state.

\section{A. Ultra Fast Single-Site Excitation}

Let us start with studying an ultra fast excitation of a single site of the complex. This is an often applied but rather artificial initial condition, as it cannot be created by light, both due to spatial (wavelength) and spectral reasons. It is important to realize that in a system of coupled chromophore, such as FMO, a single site represents a linear combination of the eigenstates of the complex, and therefore its excitation induces a coherent dynamics. This subsection discusses the established fact that an instantaneous excitation of a spatially localized state in an excitonically coupled aggregate induces dynamic coherence. This fact is a basis of an assumption that the energy transfer process in form of "incoherent hopping" leads on a microscopic level to dynamic coherence on individual molecules. As we have shown in Sections [V] and $\mathrm{V}$ ] even incoherent hopping has a natural time scale which is even longer than the time scale associated with excitation by Sun-light. We have also concluded that even if the hopping events were real, only the ensemble average would matter. This allows us to apply certain coarse graining in the present description of the energy transfer and to use constant transfer, relaxation and dephasing rates.

Figure 1 presents the EET dynamics in both the site and excitonic basis after instantaneous excitation of BChl 1 (A) or BChl 6 (B), with or without the presence of relaxation in FMO. As expected from the aggregate conformation, more specifically from the excitonic heterodimer formed by BChls 1 and 2, an excitation initially located on BChl 1 is transferred and shared with BChl 2 (see 

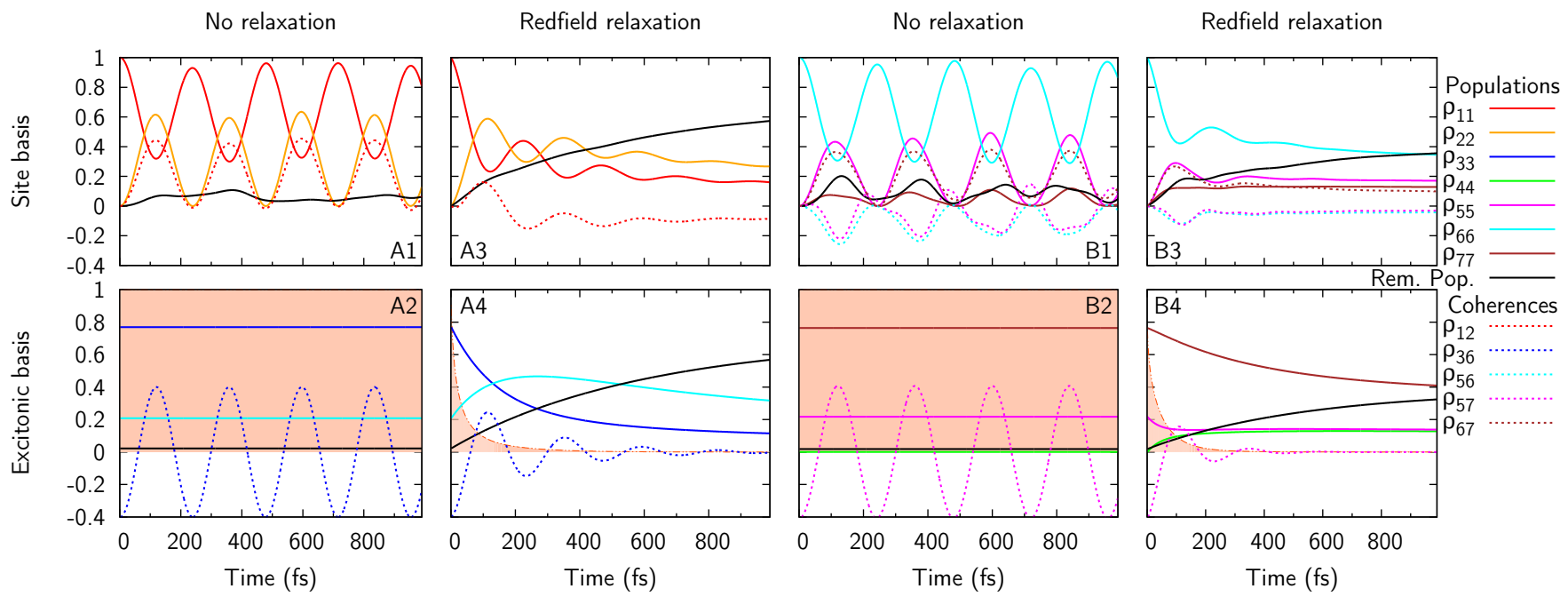

Figure 1: Time evolution of populations and coherences (diagonal and off-diagonal terms of the RDM, respectively) in the site (top) and excitonic basis (bottom) after excitation of BChl 1 (A) or 6 (B) in a free exciton system $(1 ; 2)$ or with Redfield form of relaxation $(3 ; 4)$. The shaded area represents the amount of coherence in the system.

the site basis representation, Figs. 1A1 and 1A3). Populations (diagonal elements of the reduced density matrix in a corresponding basis) display an out-of-phase evolution at the frequency $\omega_{12}=\left(E_{1}-E_{2}\right) / \hbar$, which corresponds to the frequency of the coherence evolution. In the eigenstate basis, Figs. 1A2 and 1A4, the excitation of BChl 1 populates exciton levels 3 and 6 (excitons ordered with increasing eigenenergies), which is in concordance with previous studies (e.g. [53]). We can see that the exciton populations are constant, and that only the coherences evolve phase. Comparison of the results presented in the site and excitonic basis (top vs bottom) illustrates that dynamic oscillations of the RDM off-diagonal terms, though basis dependent, are observed in both basis. When applying a Markovian dephasing in form of a secular relaxation tensor, beating can still be observed on the real part of the off-diagonal terms. However, due to the interactions with the bath, the oscillations are damped in $\sim 700 \mathrm{fs}$. This is the time scale predicted in almost all relevant work, and its achievement with a simple Redfield relaxation theory underlines the fact that the details of the relaxation theory used to describe FMO, e.g. whether Markov and secular approximations are used, is not of crucial importance.

Figure $1 \mathrm{~B}$ presents results when the excitation is initially received by $\mathrm{BChl} 6$. The relaxation pathway now involves BChls 5 and 6 and, to a smaller extend, BChl 7 (see Figs. 1B1 and1B3). Excitons 7 and 5 are the main contributors to this transfer branch (Figs. 1B2 1B4). Similarly to the previous case, an oscillatory behavior can be observed here, in both bases. Beating develops between sites 5 and 6 (site basis) and between excitons 5 and 7 (excitonic basis). With the ideal assumption of a free exciton system, undamped oscillations are observed in both basis. When considering coupling with the bath
DOF, these oscillations are quickly damped (in $\sim 400 \mathrm{fs}$ ).

Note that the off-diagonal terms of the RDM, in the local basis, stabilize around a non-zero value, but that in the basis relevant to the observation of dynamic coherences (excitonic basis), those terms quickly equal zero. This difference originates from the two distinct phenomena presented in the Introduction, namely the delocalization and the dynamic coherence. In the local basis, the non-zero values indicate delocalization of the exciton, whereas in the excitonic basis representation, no long-lived dynamic coherence survives when the system is interacting with its environment.

The amount of coherence $\Xi$ (as defined in Eq. 53) is highlighted with a shaded area to illustrate the amount of dynamic coherence in the system. As mentioned above, this quantity is experimentally relevant when used in the excitonic basis. In a free exciton system, an initially coherent system remains purely coherent and accordingly $\Xi=1$. However, the amount of coherence is damped when the system interacts with the bath DOF, and it is verified that $\Xi(t)$ follows a decay similar to the dynamic coherences.

\section{B. Excitation by Neighboring Antennae}

Now we finally consider the response of FMO excited by its neighboring chlorosome antennae. The complex starts in its ground state, and the excitation is introduced from the source $s$ (representing the neighboring BChls of the chlorosomal base plate) to the system via $\mathrm{BChl} i$ at a feeding rate $K_{f}$ such as:

$$
\frac{\partial \rho_{i i}(t)}{\partial t}=K_{f} \rho_{s s}(t)
$$





Figure 2: Time evolution of populations and coherences in the site (top) and excitonic basis (bottom) when BChl 1 (A) or 6 (B) is excited with a feeding rate of $500 \mathrm{fs}^{-1}$ in a free exciton system $(1 ; 2)$ or assuming a Redfield form of relaxation $(3 ; 4)$. The shaded area, which represents the amount of coherence in the system, shows that even without any relaxation dynamic coherences die out because of destructive interferences.

Fig. 2 shows the corresponding dynamics of EET when $\mathrm{BChl} 1$ or 6 are excited at a rate of $500 \mathrm{fs}^{-1}$. Results show that the growing population of $\mathrm{BChl} 1$ is slowly populating BChl 2 (see Figs. 2A1 and 2A3). In the excitonic basis, this corresponds to populating excitons 3 and 6 (see Figs. 2A2 and 2A4). However, even without any relaxation, this transfer does not generate a large superposition of states, as indicated from the small amplitude of the coherence oscillations observed in the excitonic basis (see Fig. 2A2). Contrary to the case where a single excitation of BChl 1 leads to a coherent dynamics (Fig. 1A2), the amount of coherence here quickly decreases with increasing populations, and it is very low when the system is significantly populated $\left(\Xi<0.04\right.$ after $\left.500 \mathrm{fs}^{-1}\right)$. This can be explained by interferences building up between coherences of excited states created at different times (which correspond to different phases) according to the feeding rate, such that: $\rho_{\alpha \beta}(t) \propto \int_{0}^{t} d \tau e^{i \omega_{\alpha \beta} / \hbar t} e^{i \phi(\tau)}$. Upon continuous feeding of BChl 1 from the neighboring antenna, the integration over different phases $\phi(\tau)$ will result in destructive interferences, which are created within a single complex and prevent any dynamic coherent superposition of excited states.

Fig. $2 \mathrm{~B}$ shows the results when excitation from the neighboring antennae is received by $\mathrm{BChl} 6$. Such a configuration results in a similar dynamics through the population of mainly BChl 5 and 6 and excitons 7 and 5 . The evolution of the amount of coherence is alike to the former case, without any creation of eigenstate superposition. Relaxation through interaction with the bath DOF will certainly not help to maintain coherent transfer, and in this case, the dynamic coherences are damped even faster $-\Xi\left(500 \mathrm{fs}^{-1}\right)<0.01$ in both excitation conditions - as shown by Figs. 2A44 and 2B 4 .
Figure 3 first presents the amount of coherence calculated according to Eq. (53) and comparison with the a measure $E$ of global entanglement defined in Ref. 60] (Fig. 33A)

$$
E=-\sum_{\alpha} \rho_{\alpha \alpha} \ln \rho_{\alpha \alpha}-S(\rho),
$$

where $S(\rho)=-\operatorname{tr}\{\rho \ln \rho\}$ is the von Neumann entropy of the state $\rho$. Also we present the amount of coherence $\Xi$ for different excitation conditions (site 1 or 6 with various feeding rates) in a free exciton system (Figs. $3 \mathrm{~B}$ and $3 \mathrm{C}$, respectively) or with relaxation (insets of Figs. $3 \mathrm{~B}$ and 3C). Fig. 3A shows that, although the different measures of coherence provide very different values in the system at initial time (very low populations), after a transient effect and once the population of the excited site becomes significant $\left(\sim 500 \mathrm{fs}^{-1}\right.$ here $)$, both models clearly show that no dynamic coherence survive, independently of the site excitation and dissipation model.

Figures $3 \mathrm{~B}$ and $3 \mathrm{C}$ show that, in a free exciton system, the amount of coherence present in a significantly populated system strongly depends upon the excitation rate. Independently of the excited site (BChl 1 or 6), dynamic coherences are seen to be created only from ultra fast excitation (faster than $\sim 100 \mathrm{fs}^{-1}$ ), i.e. when the transfer time is shorter that the period of the coherent oscillations in the acceptor system. However, for slower feeding rate which is representative of the natural excitation received from the neighboring antennae (typically $1 \mathrm{ps}^{-1}$ ) [61, 62], the constant rate feeding of an excitation to a site does not create any coherent superposition of excited states. As expected, coupling to the bath generates dephasing and results in an even smaller amount of coherence than in a free relaxation system, as shown by the insets of Figs. 

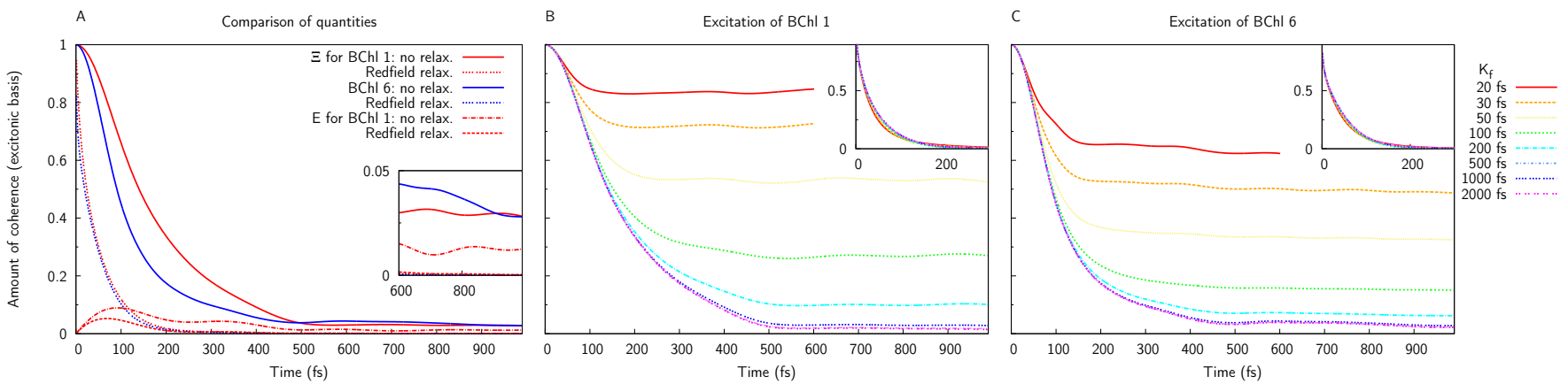

Figure 3: Evolution of the amount of coherence. (A) Comparison of the amount of coherence $\Xi$ of Eq. (53) and the quantity $E$ of Eq. (55) with an excitation rate of $500 \mathrm{fs}^{-1}$ (the inset shows a detail of the results at long time). (B and C) Amount of coherence for different excitation rates of $\mathrm{BChl} 1$ and $\mathrm{BChl} 6$, respectively in a free exciton system. The insets show results using a Redfield type of relaxation. It is shown that excitation from neighboring antennae (feeding rate of tens of picoseconds) can not lead to a coherent superposition of excited states.

3B and $3 \mathrm{C}$. Since excitation with feeding rates representative of the natural excitation (tens of picoseconds) does not lead to a superposition of coherent state already in a relaxation-free system, it is clear that the use of the Markovian dynamics does not limit our results. This is in agreement with Ref. [63]. In other words, a system interacting with a slowly relaxing bath supporting nonMarkovian evolution of excitons would, under excitation from neighboring antennae, also not lead to a coherent dynamics, which is in contrast with the concern recently raised in Ref. [64].

In our demonstration calculation we have not treated non-secular effects. Recent experimental observations suggest that non-secular effects, through the coupling of population with coherence terms, could generate dynamic coherences [19]. It has been analytically shown [45] that non-secular effect could indeed be responsible for coherent dynamics but only in the very short transient interval time following excitation. Here, even if coherences would be created, the continuous excitation from the neighboring antennae would lead to destructive interferences and suppress any coherent superposition.

\section{CONCLUSIONS}

In this paper we clarify the difference between the two effects often related to as quantum coherence. We suggest that only the eigenstate delocalization influences the yield and efficiency of excited state energy transfer in molecular aggregates, while the dynamic quantum coherence is an artifact of the particular laboratory excitation conditions. We find that in classical systems one can naturally view the slow external driving process as an integrated action of individual sudden excitations. One can represent a real classical system by a virtual ensemble of identical systems experiencing these sudden excitations, and it is likely that physical representations of such virtual ensembles may exist for some class of problems.

When attempting a similar representation of a quan- tum mechanical dynamics of an open molecular system interacting weakly with light or a neighboring molecular system, we find that a representation by sudden excitations is not satisfactory for field with finite spectral width and for neighboring antennae with finite emission spectrum width. Although the spectral width of the light defines a corresponding natural time scale of the excitation event, it is not possible to decompose the interaction with the field or a neighboring system into excitation events by some unique way. Because the yield of photosynthesis is an intrinsically averaged quantity, one can make an almost trivial observation that, in second-order response description, no matter how the quantum expectation value is decomposed, whether into some average-like contributions of individual antennae or into contributions exhibiting dynamic coherence on a microscopic level, the average remains the same. The dynamic coherence would have to be present in this average in order to be of relevance. We propose a simple experiment which could in principle test the relevance of the short time-scale of excitation events.

For a model system with important parameters taken from the most frequently studied molecular antenna exhibiting long living coherence, the FMO complex, we demonstrate that under its natural timescale of excitation no significant presence of dynamic coherence can be found, even if the system itself could avoid all the dephasing induced by its protein environment. Although we believe that the description of the excitation process represented by the density matrix is correct even on the level of individual molecules, without any subdivision of the excitation fields, the contrary would nevertheless lead to the same result for the efficiency of photosynthesis, and to the same conclusion, namely, that the dynamic coherence is not relevant for the yield of photosynthesis. The time evolution of the dynamic coherence generated by short excitation in photosynthetic systems can provide us with invaluable information about the internal dynamics of the photosynthetic energy transfer, and it can thus serve as an important diagnostic tool. However, the sug- 
gested significance of the dynamic coherence for natural light-harvesting cannot be currently experimentally con- firmed, nor can it be theoretically expected based on $\mathrm{t}$ quantum mechanics.
[1] T. Förster, in Modern Quantum Chemistry: Istanbul Lectures - Part III, Action of Light and Organic Crystals, edited by O. Cinanogu (Academic, New York, 1965), Chap. Delocalized Excitation and Excitation Transfer, pp. 93-137.

[2] R. Knox, in Excitation energy transfer and migration: theoretical considerations, edited by Govindjee (Academic, New York, 1975), pp. 183-221.

[3] G. D. Scholes, Annu. Rev. Phys. Chem. 54, 57 (2003).

[4] S. Jang, Y.-C. Cheng, D. R. Reichman, and J. D. Eaves, J. Chem. Phys. 129, 101104 (2008).

[5] T. Brixner, I. V. Stiopkin, and G. R. Fleming, Opt. Lett. 29, 884 (2004).

[6] M. L. Cowan, J. P. Ogilvie, and R. J. D. Miller, Chem. Phys. Lett. 386, 184 (2004).

[7] T. Brixner, T. Mančal, I. V. Stiopkin, and G. R. Fleming, J. Chem. Phys. 121, 4221 (2004).

[8] A. V. Pisliakov, T. Mančal, and G. R. Fleming, J. Chem. Phys. 124, 234505 (2006).

[9] G. S. Engel, T. R. Calhoun, E. L. Read, T.-K. Ahn, T. Mančal, Y.-C. Cheng, R. E. Blankenship, and G. R. Fleming, Nature 446, 782 (2007).

[10] V. I. Prokhorenko, A. R. Holzwarth, F. R. Nowak, and T. J. Aartsma, J. Phys. Chem. B 106, 9923 (2002).

[11] H. Lee, Y.-C. Cheng, and G. Fleming, Science 316, 1462 (2007).

[12] T. R. Calhoun, N. S. Ginsberg, G. S. Schlau-Cohen, Y.C. Cheng, M. Ballottari, R. Bassi, and G. R. Fleming, J. Phys. Chem. B 113, 16291 (2009).

[13] I. P. Mercer, Y. C. El-Taha, N. Kajumba, J. P. Marangos, J. W. G. Tisch, M. Gabrielsen, R. J. Cogdell, E. Springate, and E. Turcu, Phys. Rev. Lett. 102, 057402 (2009).

[14] E. Collini, C. Y. Wong, K. E. Wilk, P. M. G. Curmi, P. Brumer, and G. D. Scholes, Nature 463, 644 (2010).

[15] E. Harel and G. S. Engel, Proc. Natl. Acad. Sci. U. S. A. 109, 706 (2012).

[16] E. Collini and G. D. Scholes, Science 323, 369 (2009).

[17] I. Kassal, J. Yuen-Zhou, and S. Rahimi-Keshari, J. Phys. Chem. L. 4, 362 (2013).

[18] D. B. Turner, R. Dinshaw, K. Lee, M. S. Belsley, K. E. Wilk, P. M. G. Curmi, and G. D. Scholes, Phys. Chem. Chem. Phys. 14, 4857 (2012).

[19] G. Panitchayangkoon, D. Hayes, K. A. Fransted, J. R. Caram, E. Harel, J. Wen, R. E. Blankenship, and G. S. Engel, Proc. Natl. Acad. Sci. U. S. A. 107, 12766 (2010).

[20] G. Panitchayangkoon, D. V. Voronine, D. Abramavicius, J. R. Caram, N. H. C. Lewis, S. Mukamel, and G. S. Engel, Proc. Natl. Acad. Sci. U. S. A. 108, 20908 (2011).

[21] L. A. Pachón and P. Brumer, J. Phys. Chem. Lett. 2, 2728 (2011).

[22] C. Kreisbeck and T. Kramer, J. Phys. Chem. Lett. 3, 2828 (2012).

[23] B. Hein, C. Kreisbeck, T. Kramer, and M. Rodríguez, New J. Phys. 14, 023018 (2012).

[24] T. Fujita, J. Huh, S. K. Saikin, J. C. Brookes, and A. Aspuru-Guzik, ArXiv 1304.4902, (2013).
[25] M. H. Cho, H. M. Vaswani, T. Brixner, J. Stenger, and G. R. Fleming, J. Phys. Chem. B 109, 10542 (2005).

[26] M. Mohseni, P. Rebentrost, S. Lloyd, and A. AspuruGuzik, J. Chem. Phys. 129, 174106 (2008).

[27] P. Rebentrost, M. Mohseni, I. Kassal, S. Lloyd, and A. Aspuru-Guzik, New J. Phys. 11, 033003 (2009).

[28] D. Hayes, G. Panitchayangkoon, K. A. Fransted, J. R. Caram, J. Wen, K. F. Freed, and G. S. Engel, New J. Phys. 12, 065042 (2010).

[29] A. Ishizaki and G. R. Fleming, New Journal of Physics 12, 055004 (2010).

[30] J. Wu, F. Liu, Y. Shen, J. Cao, and R. J. Silbey, New J. Phys. 12, 105012 (2010).

[31] D. Abramavicius and S. Mukamel, J. Chem. Phys. 134, 174504 (2011).

[32] J. Strümpfer and K. Schulten, J. Chem. Phys. 134, 095102 (2011).

[33] F. Caycedo-Soler, A. W. Chin, J. Almeida, S. F. Huelga, and M. B. Plenio, J. Chem. Phys. 136, 155102 (2012).

[34] J. R. Caram, N. H. C. Lewis, A. F. Fidler, and G. S. Engel, J. Chem. Phys. 136, 104505 (2012).

[35] C. Olbrich and U. Kleinekathöfer, J. Phys. Chem. B 114, 12427 (2010).

[36] S. Shim, P. Rebentrost, S. Valleau, and A. Aspuru-Guzik, Biophys. J. 102, 649 (2012).

[37] N. Christensson, H. F. Kauffmann, T. Pullerits, and T. Mančal, J. Phys. Chem. B 116, 7449 (2012).

[38] V. Tiwari, W. K. Peters, and D. M. Jonas, Proc. Natl. Acad. Sci. U. S. A. 110, 1203 (2013).

[39] A. Chenu, N. Christensson, H. F. Kauffmann, and T. Mančal, Scientific Report 3, 2029 (2013).

[40] A. W. Chin, J. Prior, R. Rosenbach, F. Caycedo-Soler, S. F. Huelga, and M. B. Plenio, Nature Physics 9, 113 (2013).

[41] Y.-C. Cheng and G. R. Fleming, Annu. Rev. Phys. Chem. 60, 241 (2009).

[42] X.-P. Jiang and P. Brumer, J. Chem. Phys. 94, 5833 (1991).

[43] P. Brumer and M. Shapiro, Proc. Nat. Am. Soc. 109, 19575 (2012).

[44] T. Mančal and L. Valkunas, New Journal of Physics 12, 065044 (2010).

[45] L. A. Pachón and P. Brumer, ArXiv 1210.6374v1 (2012).

[46] A. Ishizaki and G. R. Fleming, J. Phys. Chem. B 115, 6227 (2011).

[47] S. Mukamel, Principles of nonlinear spectroscopy (Oxford University Press, Oxford, 1995).

[48] R. Loudon, The Quantum Theory of Light, 3rd edition ed. (Oxford University Press, Oxford, 2000).

[49] L. Valkunas, D. Abramavičius, and T. Mančal, Molecular Excitation Dynamics and Relaxation: Quantum Theory and Spectroscopy (Wiley-VCH, Weinheim, 2013).

[50] D. Manzano, PLOSone 8, 57041 (2013).

[51] K. Hoki and P. Brumer, in Procedia Chemistry (Elsevier, 22nd Solvay Conference on Chemistry, 2011), Vol. 3, pp. $122-131$.

[52] M. T. W. Milder, B. Brüggemann, R. van Grondelle, and 
J. L. Herek, Photosynth. Res. 104, 257 (2010).

[53] J. Adolphs and T. Renger, Biophys. J. 91, 2778 (2006).

[54] A. Ben-Shem, F. Frolow, and N. Nelson, FEBS lett. 564, 274 (2004).

[55] D. Tronrud, J. Wen, L. Gay, and R. Blankenship, Photosynth. Res. 100, 79 (2009).

[56] C. Olbrich, J. Strümpfer, K. Schulten, and U. Kleinekathöfer, J. Phys. Chem. Lett. 2, 1771 (2011).

[57] J. Wen, H. Zhang, M. L. Gross, and R. E. Blankenship, Proc. Natl. Acad. Sci. U. S. A. 106, 6134 (2009).

[58] V. May and O. Kühn, Charge and Energy Transfer Dynamics in Molecular Systems (Wiley-VCH, Berlin, 2001).

[59] A. Ishizaki and G. R. Fleming, Proc. Natl. Acad. Sci. U.
S. A. 106, 17255 (2009).

[60] M. Sarovar, A. Ishizaki, G. R. Fleming, and K. B. Whaley, Nature Physics 6, 462 (2010).

[61] S. Savikhin, Y. Zhu, S. Lin, R. E. Blankenship, and W. S. Struve, J. Chem. Phys. 10322, 10322 (1994).

[62] J. Martiskainen, J. Linnanto, R. Kananavičius, V. Lehtovuori, and J. Korppi-Tommola, Chem. Phys. Lett. 477, 216 (2009).

[63] S. Jesenko and M. Žnidarič, J. Chem. Phys. 138, 174103 (2013).

[64] F. Fassioli, A. Olaya-Castro, and G. D. Scholes, J. Phys. Chem. Lett. 3, 3136 (2012). 